\title{
Design, Characterization and In-vitro Study of Folic Acid Conjugated-Chitosan Functionalized Gold Nanoparticles for Targeted Delivery of 5-Fluorouracil in Breast Cancer
}

\author{
Akinyelu Jude and Moganavelli Singh
}

\begin{abstract}
This present investigation explores the use of 5-fluorouracil (5-FU) encapsulated gold nanoparticles (GNP), functionalized with Folic acid (FA) conjugated Chitosan (CS) (FA-CS-GNP-5-FU) for improved drug efficacy with minimal side effects. Drug-excipient interaction was achieved by ionic complexation and drug encapsulation efficiency was appropriately determined. The physio-chemical properties of the NPs were investigated, in vitro drug release pattern and in vitro cytotoxicity were evaluated on cancer and normal cells. The results reveal that the obtained nanoparticles were monodispersed with suitable average sizes and positive surface charges. Also, the drug was easily encapsulated by the functionalized NPs and displayed a $\mathbf{p H}$ dependent and sustained drug release profile. Compared with the free drug, FA-CS-GNP-5-FU displayed higher anticancer activity against breast cancer cells which had high expression of folate receptors. Thus, FA-CS GNP-5-FU provide a potential way to enhance the efficiency of anti-tumour drugs by folate receptor mediated targeting delivery.
\end{abstract}

Keywords - Gold nanoparticles, folate-conjugated chitosan.

\section{INTRODUCTION}

Optimization of the pharmacological potential of a drug with reduction in the adverse effects is the major pre-requisite for a drug delivery system. The use of functionalized nanoparticles (NP) can provide site specific or targeted delivery along with optimal drug release. The use of gold nanoparticles (GNPs) has increasingly become popular owing to its inherent optical features, relative non-toxicity and suitable biocompatibility [1]. Chitosan (CS) is a non-toxic biocompatible polymer that can effectively functionalise and stabilise GNPs in an aqueous solution. CS has an amino and hydroxyl group which can serve as chelating sites for a variety of compounds. 5-fluorouracil (5-FU), a thymidylate synthase inhibitor, is extensively used in the treatment of solid tumours. However, poor bioavailability, short plasma half-life coupled with off-target cytotoxicity have limited its use in chemotherapy. To improve the therapeutic efficacy of 5-FU with minimal side effects, it can be selectively

Akinyelu Jude, PhD Student, University of KwaZulu-Natal, Westville, Durban, South Africa.

Moganavelli Singh, University of KwaZulu-Natal, Westville, Durban, South Africa. delivered to target sites using specifically designed carriers [2]. Further, the folate receptor which is highly expressed in various human cancers can be targeted for improved receptor-mediated uptake of chemotherapeutics. Folic acid is widely used as a ligand for folate receptor because they are cheap, nontoxic, retain high affinity for folate receptors, and are stable in the systemic circulation [3]. In the present investigation, 5-FU was encapsulated by GNPs, and further functionalized with Folic acid conjugated chitosan, for the evaluation of anticancer activity against human breast cancer cells (MCF-7) and normal cells (HEK-293).

\section{RESULTS AND DISCUSSION}

\section{A. UV-vis spectroscopy, Particle morphology, sizing, and zeta potential analysis}

The reduction of gold chloride to form GNPs, was visually confirmed, as the colloidal solution changed to wine red colour within 15 mins. Direct proof of GNP's synthesis was obtained from UV-vis absorption spectra of GNP solution (Fig. 1A), which shows a defined absorption peak in the wavelength range of 522-525 nm. Also, FA-CS-GNP-5-FU with an encapsulation efficiency of $\sim 79 \%$, showed well-defined absorption bands in similar wavelength range. A peak in this range is generally attributed to the surface plasmon excitation of small spherical GNPs [1]. However, a decline in the absorption intensity was observed for FA-CS-GNP and FA-CS-GNP-5-FU, which may be indicative of a change in GNP's surface upon the adsorption of composites. The formation of nanocomposites was further corroborated by TEM analysis. TEM images as depicted in Fig. 1B-D, reveal that most of the blank GNPs were spherically shaped with an average size of $29 \mathrm{~nm}$. Similar shapes were displayed FA-CS-GNP and FA-CS-GNP-5-FU, however, with an increased average sizes $31 \mathrm{~nm}$ and $33 \mathrm{~nm}$ respectively. The hydrodynamic sizes of blank GNPs, FA-CS-GNP and FA-CS-GNP-5-FU as measured by Nanoparticle tracking analyser were $\sim 65.9 \mathrm{~nm}, \sim 115 \mathrm{~nm}$ and $\sim 144 \mathrm{~nm}$ respectively. This was bigger as compared to the corresponding sizes via TEM measurements. This could be attributed to the swelling of particles in aqueous medium during hydrodynamic measurements in contrast to TEM which measures sample sizes in their dry state. Also, FA-CS-GNP and FA-CS-GNP-5-FU exhibited high stability as indicated by the measured zeta 
potential values of +61.7 and $+61.1 \mathrm{mV}$ respectively, in contrast to blank GNP (-7.3mV). A shift from the negative zeta potential value of blank GNPs to a highly positive value of the drug carrier is an evidence of the functionalization of GNP by FA-CS.

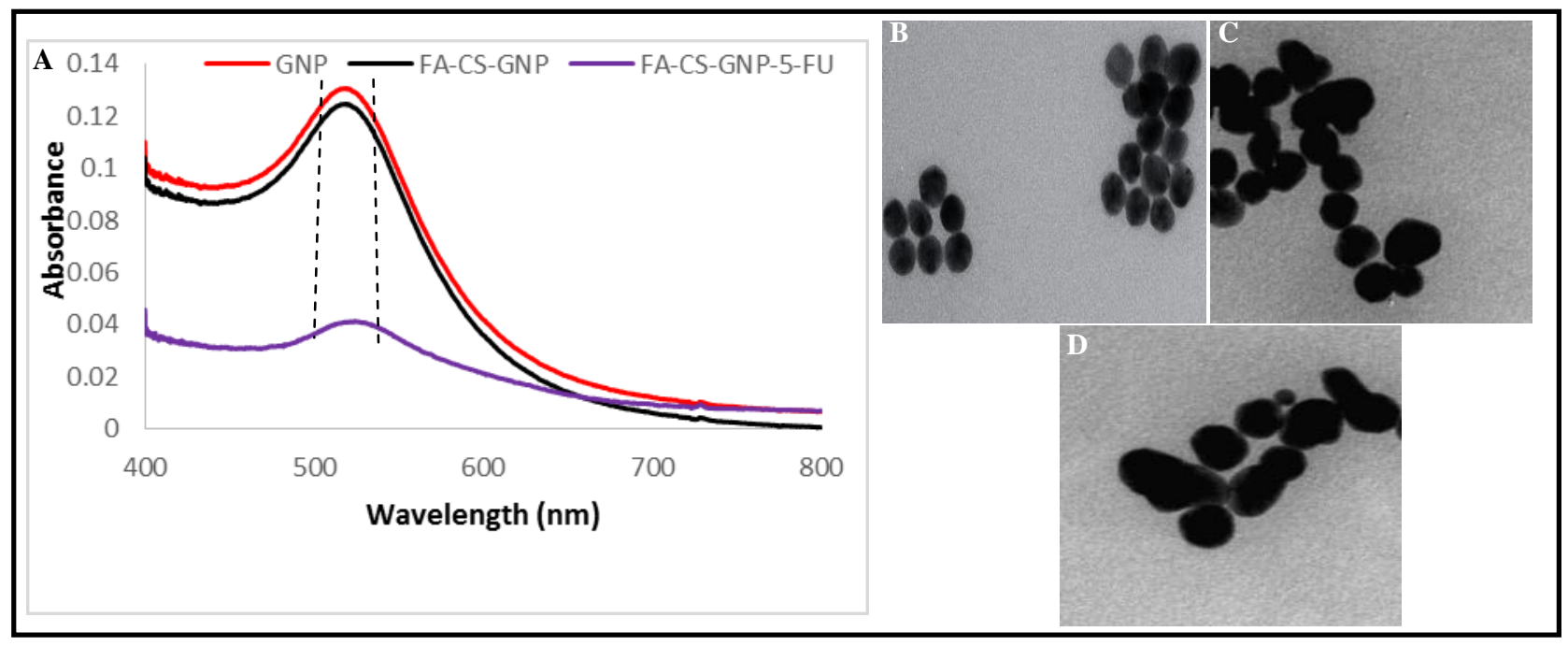

Fig.1 showing [A] UV-vis spectra of GNP, FA-CS-GNP, and FA-CS-GNP-5-FU, [B-D] TEM images of GNP, FA-CS-GNP, and FA-CS-GNP-5-FU, respectively

$70 \%$ of 5-FU was released from FA-CS-GNP-5-FU at $\mathrm{pH} \sim 5$

\section{B. FT-IR Analysis}

Fig. 2 depicts the FT-IR spectrum of the composites. The presence of characteristic bands of CS $(\sim 1700 \mathrm{~cm}-1, \sim 3484$ $\mathrm{cm}-1)$ and FA ( 1480 $-1700 \mathrm{~cm}-1)$ with minor shift in FA-CS-GNP-5-FU, confirms the presence of FA-CS on the synthesised drug encapsulated GNP. All peaks assignment were done according to literature [4,5].

\section{In-vitro drug release}

The release of 5-FU from the FA-CS-GNP-5-FU was dependent on $\mathrm{pH}$ of the medium (Fig. 3). After $72 \mathrm{hrs}, 92 \%$ and and $\sim 7.4$ respectively. Thus, it can be understood that the carriers showed faster drug release at low $\mathrm{pH}$ than neutral $\mathrm{pH}$. Therefore, it is conceivable that the acidic tumour environment might trigger the release of 5-FU from the synthesised carriers. The relatively reduced release of the drug from the nanocomposite at $\mathrm{pH} 7.44$ might ensure decreased drug toxicity to normal tissues. Importantly, the sustained drug release profile from the carriers over a long duration, would minimize the frequency of dosing intervals. Previous investigations demonstrated the sustained release of 5-FU from cationic modified inorganic nanoparticles at similar $\mathrm{pH}$ regions [1].

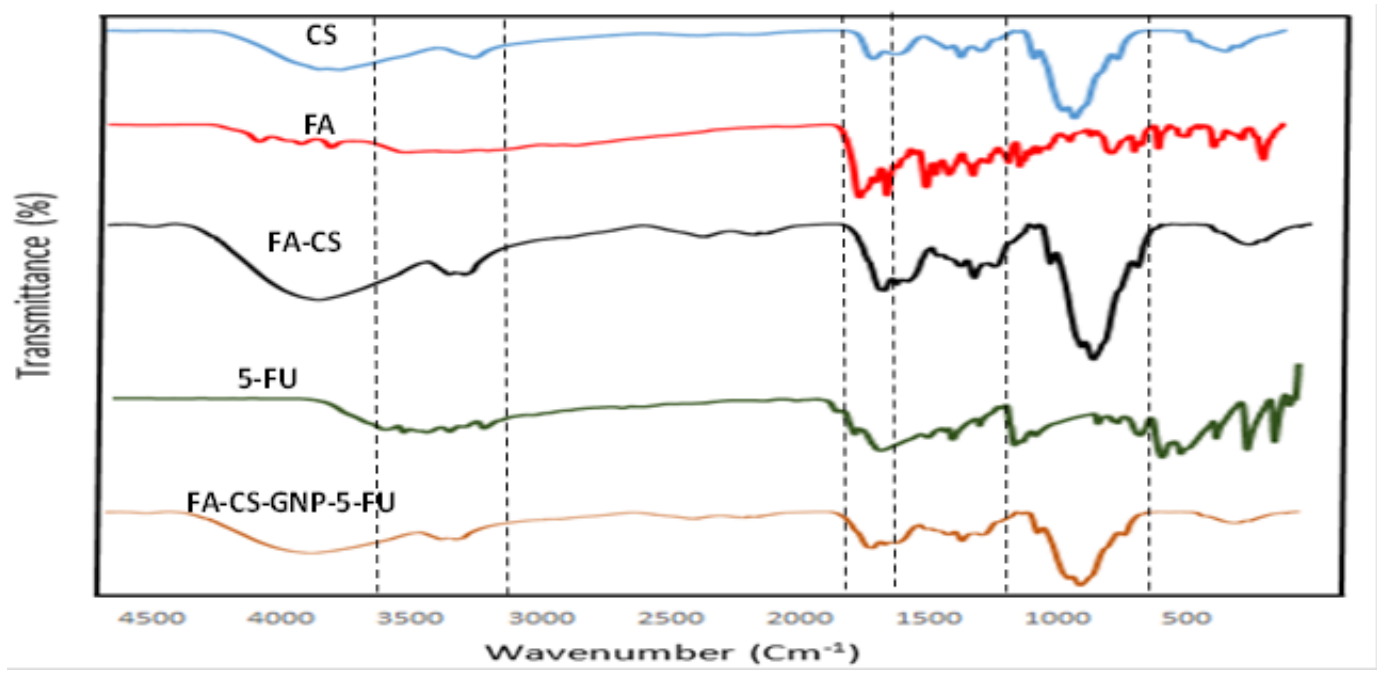

Fig.2 showing FT-IR spectral analysis of CS, FA, FA-CS, free 5-FU and FA-CS-GNP-5-FU 


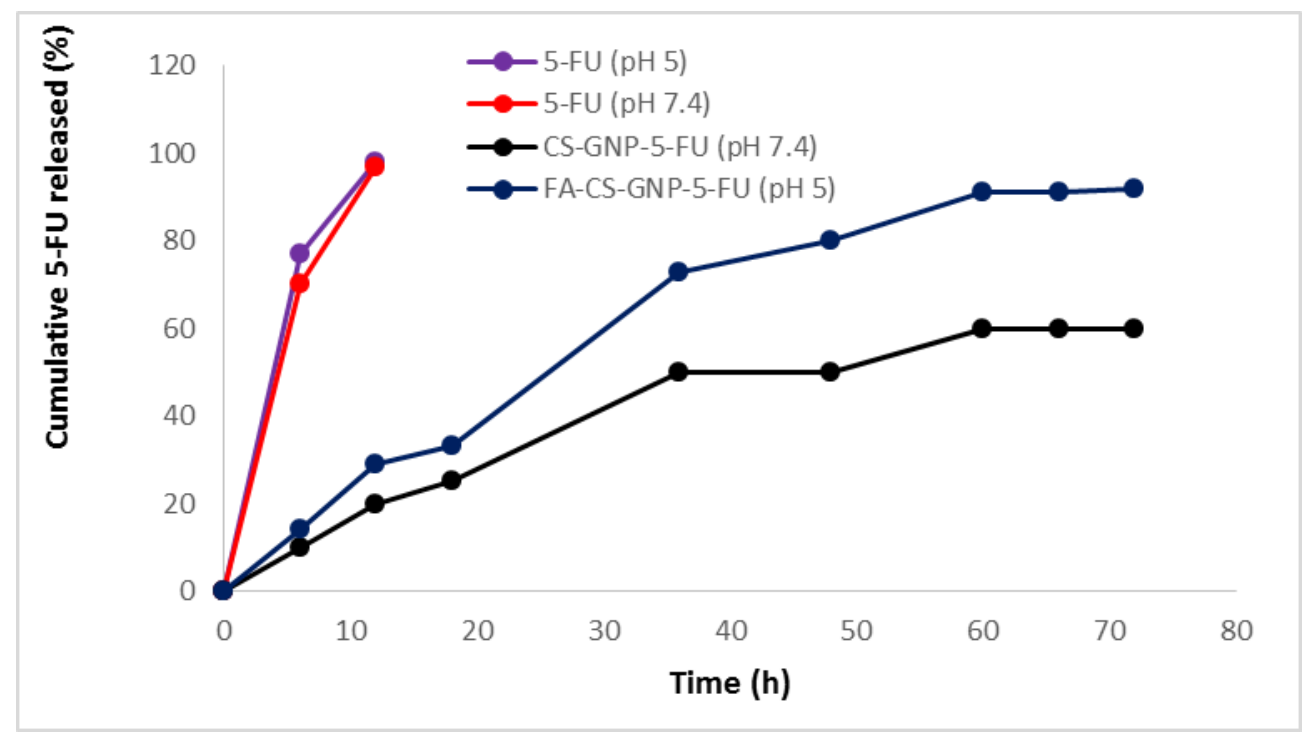

Fig.3 showing drug release profile of 5-FU encapsulated nanocomposite

\section{Evaluation of cytotoxicity}

Our results as represented in Fig.4, show relative non-toxic effects of FA-CS-GNP on all the investigated cell lines. Hence, it can be deduced that any cytotoxic effect is not due to the drug free carrier. Further, FA-CS-GNP-5-FU (Fig. 4A) showed a dose-dependent increase in cytotoxicity against MCF-7 cells. The half maximal inhibitory (IC50) values for FA-CS-GNP-5-FU on MCF-7 cells was $20 \mu \mathrm{g} / \mathrm{mL}$ while free 5-FU at an identical dose resulted in $<30 \%$ cell death. The improved cytotoxicity shown by FA-CS-GNP-5-FU on MCF-7 cells compared to free 5-FU, may be due to the controlled release of 5-FU and the ability of GNPs to evade cellular barriers, thereby, leading to an increased 5-FU accumulation in the cancer cells. Also, the functionalization of GNP with
FA-CS, may have induced specific binding of GNPs to the folate receptors $(F R \alpha)$ which are highly expressed on the MCF-7 cell surface. This interaction may have facilitated folate receptor mediated endocytosis, resulting in enhanced cellular uptake of FA-CS-GNP-5-FU by the cells. The receptor mediated internalization of FA-CS-GNP-5-FU was further demonstrated in a competition assay, using free FA as the competitor. Results show a significant decline in the anticancer efficacy of various amount of FA-CS-GNP-5-FU on the MCF-7 cells pre-incubated with FA. Interestingly, the viability of HEK-293 cells (Fig.4B) at the highest studied concentration of $40 \mu \mathrm{g} / \mathrm{ml}$ of FA-CS-GNP-5FU was $80 \%$, which clearly suggests its minimal toxicity to surrounding normal cells

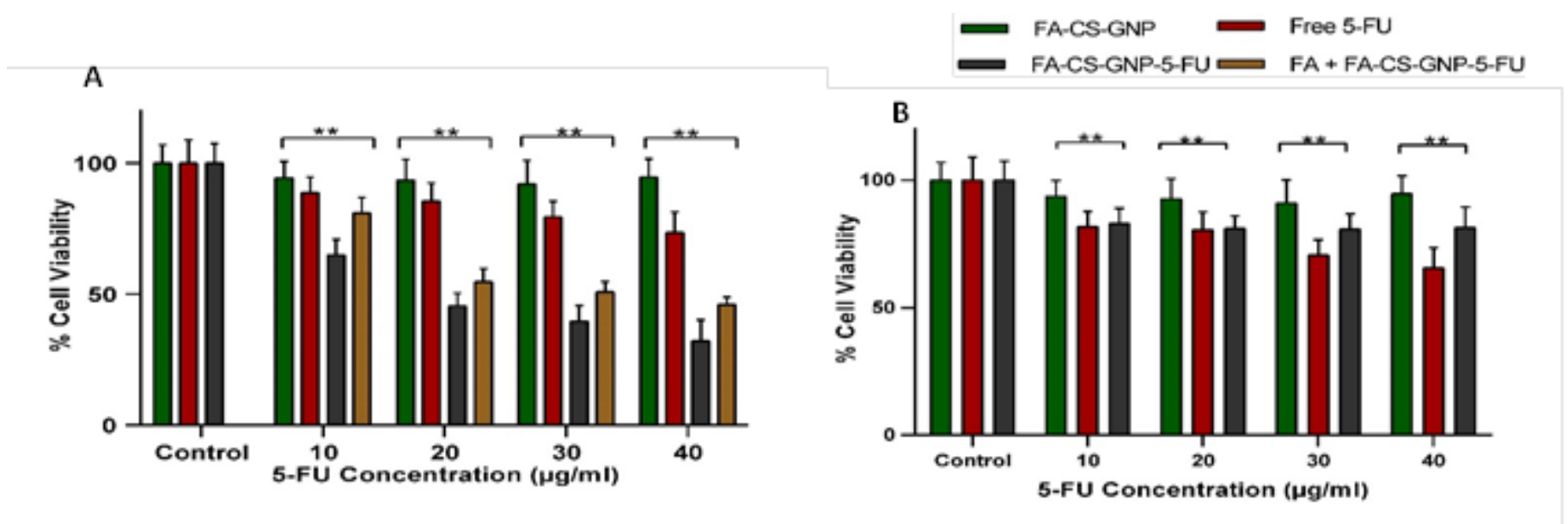

Fig. 4 In-vitro Cytotoxicity analysis of FA-CS-GNP-5-FU towards [A] MCF-7, [B] HEK-293 cells. (**P < 0.01 was considered statistically significant, mean $\pm \mathrm{SD} ; \mathrm{n}=3$ )

\section{CONCLUSION}

A small and highly stable carrier of 5-FU was synthesized. Cell toxicity assay proved that FA-CS-GNP-5-FU had better efficacy than free 5-FU, which might reduce the dosage regimen and subsequent side effects. These results confirm the potential of FA-CS functionalized GNPs as an effective delivery platform for 5-FU or similar chemotherapeutic agents. 


\section{ACKNOWLEDGMENT}

The authors acknowledge the funding received from National Research foundation, South Africa (NRF) and the world Academy of Sciences, Italy (TWAS).

\section{REFERENCES}

[1] M. Safwat et al., "Gold nanoparticles enhance 5-fluorouracil anticancer against colorectal cancer cells" Int. J. pharmaceutics., vol. 513, pp. 648-658, 2016.

https://doi.org/10.1016/j.ijpharm.2016.09.076

[2] O. Udofot et al., "Cytotoxicity of 5-fluorouracil-loaded pH-sensitive liposomal nanoparticles in colorectal cancer cell lines, ". Integr Cancer Sci Ther, vol 2, pp. 245, 2015 https://doi.org/10.15761/ICST.1000150

[3] N. Dhas et al., "Design, optimization and in-vitro study of folic acid conjugated-chitosan functionalized PLGA nanoparticle for delivery of bicalutamide in prostate cancer" Powder Tech., vol. 283, pp. 234-245, 2015

https://doi.org/10.1016/j.powtec.2015.04.053

[4] M. Sharma et al., "Design of folic acid conjugated chitosan nano-cur-bio enhancers to attenuate the hormone-refractory metastatic prostate carcinoma by augmenting oral bioavailability". RSC Adv., vol. 6, pp. 25137-25148, 2016 https://doi.org/10.1039/C5RA17599B

[5] AEB Yassin et al., "Optimization of 5-flurouracil solid-lipid nanoparticles: a preliminary study to treat colon cancer". Int $J$ Med Sci.,vol 7, pp. 398, 2010

https://doi.org/10.7150/ijms.7.398 